PROCEEDINGS OF THE

AMERICAN MATHEMATICAL SOCIETY

Volume 133, Number 7, Pages 2051-2059

S 0002-9939(05)07735-X

Article electronically published on January 14, 2005

\title{
A *-CLOSED SUBALGEBRA OF THE SMIRNOV CLASS
}

\author{
STEPHAN RAMON GARCIA
}

(Communicated by Joseph A. Ball)

\begin{abstract}
We study real Smirnov functions and investigate a certain $*$-closed subalgebra of the Smirnov class $N^{+}$containing them. Motivated by a result of Aleksandrov, we provide an explicit representation for the space $H^{p} \cap \overline{H^{p}}$. This leads to a natural analog of the Riesz projection on a certain quotient space of $L^{p}$ for $p \in(0,1)$. We also study a Herglotz-like integral transform for singular measures on the unit circle $\partial \mathbb{D}$.
\end{abstract}

\section{INTRODUCTION}

This note concerns a certain $*$-closed subalgebra of the Smirnov class and several of its applications. We first require a few preliminaries on the classical theory of Hardy spaces, most of which can be found in [4.

For each $p \in(0, \infty)$, the Hardy space $H^{p}$ is the space of analytic functions $f$ on the unit disk $\mathbb{D}$ for which the quantity

$$
\|f\|_{p}:=\sup _{0<r<1}\left(\frac{1}{2 \pi} \int_{-\pi}^{\pi}\left|f\left(r e^{i t}\right)\right|^{p} d t\right)^{\frac{1}{p}}
$$

is finite. If $p \in[1, \infty)$, then $H^{p}$ is a Banach space with norm $\|\cdot\|_{p}$. For $p \in(0,1)$, $H^{p}$ is not normable, although the quantity

$$
d\left(f_{1}, f_{2}\right):=\left\|f_{1}-f_{2}\right\|_{p}^{p}
$$

defines a translation-invariant metric with respect to which $H^{p}$ is complete. We now briefly summarize the inner-outer factorization of $H^{p}$ functions.

An outer function is a function $F$ of the form

$$
F(z)=e^{i \gamma} \exp \left[\frac{1}{2 \pi} \int_{-\pi}^{\pi} \frac{e^{i t}+z}{e^{i t}-z} u\left(e^{i t}\right) d t\right]
$$

where $\gamma$ is a real constant and $u$ is a real-valued function in $L^{1}$ of the unit circle $\partial \mathbb{D}$. The expression in square brackets is called the Herglotz integral of $u$. Since $u=\log |F|$ a.e. on $\partial \mathbb{D}$, an outer function is determined up to a unimodular constant factor by its modulus on $\partial \mathbb{D}$.

An inner function is an $H^{\infty}$ function $\varphi$ that satisfies $|\varphi|=1$ a.e. on $\partial \mathbb{D}$. Here $H^{\infty}$ denotes the space of bounded analytic functions $f$ on $\mathbb{D}$ equipped with the supremum norm. Any inner function $\varphi$ factors as $\varphi=e^{i \gamma} B S$ where $\gamma$ is a real

Received by the editors February 3, 2004 and, in revised form, March 5, 2004.

2000 Mathematics Subject Classification. Primary 30D55.

Key words and phrases. Riesz projection, Smirnov class, Herglotz integral, singular measure, Beurling's theorem, backward shift, pseudocontinuation. 
constant, $B$ is a Blaschke product and $S$ is a singular inner function (see [4 for definitions).

Each $H^{p}$ space is contained in the Smirnov class $N^{+}$, which consists of all functions of the form $h_{1} / h_{2}$ where $h_{1}, h_{2}$ belong to $H^{\infty}$ and $h_{2}$ is outer. Each $f$ in $N^{+}$possesses nontangential limiting values almost everywhere on $\partial \mathbb{D}$ and factors (uniquely up to unimodular constant factors) as $f=I_{f} F$ where $I_{f}$ is an inner function and $F$ is an outer function.

A function $f$ belonging to the Smirnov class $N^{+}$is called a real Smirnov function if its boundary function is real-valued almost everywhere on $\partial \mathbb{D}$. The set of all real Smirnov functions, denoted by $R^{+}$, is a real subalgebra of $N^{+}$and was explicitly described by Helson [7]. He showed that if $\psi_{1}$ and $\psi_{2}$ are relatively prime inner functions such that $\psi_{1}-\psi_{2}$ is outer, then the function

$$
f=i \frac{\psi_{1}+\psi_{2}}{\psi_{1}-\psi_{2}}
$$

is a real Smirnov function and every real Smirnov function arises this way. Although elegant, this representation has its limitations. For example, the inner functions $\psi_{1}$ and $\psi_{2}$ are often difficult to identify for a given $f$ and there are no general criteria describing when the difference of inner functions is outer.

We are concerned with real Smirnov functions and their applications. We first establish a practical description of $R^{+}$in Section 2, extending a result of Sarason and the author [6]. In Section 3 we discuss the subalgebra $R^{+}+i R^{+}$of $N^{+}$, noting briefly that it can be used to obtain an explicit analog of Beurling's theorem for the backward shift operator on $H^{p}$ for $p \in[1, \infty)$. We discuss two other applications of this algebra in Sections 4 and 5 . Motivated by a result of Aleksandrov 11, we provide an explicit description of the space $H^{p} \cap \overline{H^{p}}$. This leads to a natural analog of the Riesz projection on a certain quotient space of $L^{p}$ for $p \in(0,1)$. In Section 5 we study a Herglotz-like integral transform for singular measures on $\partial \mathbb{D}$.

The author wishes to thank Donald Sarason for his comments and suggestions regarding this note and also to acknowledge the input and advice of Mihai Putinar.

\section{Real Smirnov functions}

Let $R O$ denote the subset of outer functions in $R^{+}$. For obvious reasons, we refer to such functions as real outer functions. Let $T$ denote the linear fractional transformation

$$
T(z):=i \frac{1-i z}{1+i z} .
$$

In 6], Sarason and the author proved that any $F$ belonging to $R O$ can be represented as a locally uniformly convergent product

$$
F(z)=|F(0)| \prod_{n=1}^{\infty} \frac{T\left(\varphi_{n}^{+}\right)}{T\left(\varphi_{n}^{-}\right)}
$$

where $\varphi_{n}^{+}$and $\varphi_{n}^{-}$are inner functions naturally associated with the boundary values of $\arg F$ on $\partial \mathbb{D}$.

To describe the most general function in $R^{+}$, we require several simple observations. Note that $T=T_{-} / T_{+}$where the functions $T_{+}$and $T_{-}$are defined by

$$
T_{ \pm}(z):=\frac{1}{\sqrt{2}} \frac{1 \pm i z}{1 \pm i}
$$


and satisfy $\left\|T_{ \pm}\right\|_{\infty}=1$. Furthermore, we have

whence

$$
T_{ \pm}(z)^{2}= \pm \frac{(1 \pm i z)^{2}}{4 i}
$$

$$
T_{+}(z)^{2}+T_{-}(z)^{2}=z
$$

Now define the functions $K_{+}(z)$ and $K_{-}(z)$ by setting

$$
\begin{aligned}
K_{ \pm}(z) & :=\frac{z}{T_{ \pm}(z)^{2}} \\
& =1+T(z)^{ \pm 2},
\end{aligned}
$$

and note that $K_{ \pm}(z)=-4 k(\mp i z)$ where $k(z)=z /(1-z)^{2}$ denotes the Koebe function. The well-known properties of the Koebe function indicate that the functions $K_{ \pm}$map the open unit disk $\mathbb{D}$ onto the complement of the half-line $(1, \infty)$. Most importantly, $K_{ \pm} \geq 1$ on $\partial \mathbb{D}$.

It is easy to see that $R^{+} \cap H^{p}$ contains nonconstant functions if and only if $p \in(0,1)$. For such $p$, we define $R^{p}$ to be the real linear subspace $R^{+} \cap H^{p}$ of $H^{p}$. Let us denote the inner and outer factors of a function $f$ in $N^{+}$by $I_{f}$ and $F$ respectively. Our elementary observations can now be put to good use:

Proposition 1. If $f=I_{f} F$ belongs to $R^{+}$(resp. $R^{p}$ ), then:

(1) $K_{ \pm}\left(I_{f}\right) \geq 1$ a.e. on $\partial \mathbb{D}$ and belongs to $R^{q}$ for every $q \in\left(0, \frac{1}{2}\right)$.

(2) $f=K_{ \pm}\left(I_{f}\right)\left[T_{ \pm}\left(I_{f}\right)^{2} F\right]$.

(3) $\left|T_{ \pm}\left(I_{f}\right)^{2} F\right| \leq|F|$ a.e. on $\partial \mathbb{D}$.

(4) $T_{ \pm}\left(I_{f}\right)^{2} F$ belongs to $R O$ (resp. $R O \cap R^{p}$ ).

(5) $T_{ \pm}\left(I_{f}\right)^{2} F$ and $f$ have the same sign a.e. on $\partial \mathbb{D}$.

(6) $f=T_{+}\left(I_{f}\right)^{2} F+T_{-}\left(I_{f}\right)^{2} F$. In other words, $f$ is the sum of two functions in $R O$ (resp. $R O \cap R^{p}$ ).

Thus the general function $f=I_{f} F$ in $R^{+}$(resp. $R^{p}$ ) can be expressed as the product of a real Smirnov function $K_{ \pm}\left(I_{f}\right)$ that is bounded below by 1 a.e. on $\partial \mathbb{D}$ and an $R O$ function $T_{ \pm}\left(I_{f}\right)^{2} F$ whose boundary behavior is closely tied to that of $f$ itself. With this practical description of $R^{+}$, we can prove the following result of Helson and Sarason [8] and Neuwirth and Newman [9].

Theorem. If $f \in R^{\frac{1}{2}}$ and $f \geq 0$ a.e. on $\partial \mathbb{D}$, then $f$ is constant.

Proof. If $f$ is outer, then $f^{\frac{1}{2}}$ belongs to $R^{+} \cap H^{1}$ and is therefore constant. If $f$ has a nontrivial inner factor $I_{f}$, then Proposition 1 shows that $f$ is a nonnegative multiple of $K_{+}\left(I_{f}\right)$. Thus $T\left(I_{f}\right)^{2}=K_{+}\left(I_{f}\right)-1$ belongs to $R O \cap R^{\frac{1}{2}}$, and hence $T\left(I_{f}\right) \in R O \cap H^{1}$, a contradiction.

\section{THE SPACES $C^{p}$}

Let $C^{+}$denote the complex subalgebra $R^{+}+i R^{+}$of $N^{+}$. With respect to the translation-invariant metric

$$
\rho(f, g):=\frac{1}{2 \pi} \int_{-\pi}^{\pi} \log \left(1+\left|f\left(e^{i t}\right)-g\left(e^{i t}\right)\right|\right) d t
$$

on $N^{+}[2], C^{+}$is a complete metric space and a topological algebra.

Since the algebra of boundary functions corresponding to $C^{+}$is closed under complex conjugation, $C^{+}$carries a natural involution. Observe that if $f=a+i b$ 
where $a$ and $b$ belong to $R^{+}$, then $\widehat{f}:=a-i b$ agrees with $\bar{f}$ on $\partial \mathbb{D}$. The conjugation operator $\hat{\cdot}$ preserves outer factors since

$$
|f|^{2}=|\widehat{f}|^{2}=|a|^{2}+|b|^{2}
$$

a.e. on $\partial \mathbb{D}$. Hence we may write $f=I_{f} F$ and $\widehat{f}=I_{\widehat{f}} F$ where $F$ is an outer function and $I_{f}$ and $I_{\widehat{f}}$ are inner.

We are mainly concerned with the intersection of the algebra $C^{+}$with the spaces $H^{p}$. For each $p$, define $C^{p}:=C^{+} \cap H^{p}$ and observe that this intersection contains nonconstant functions if and only if $p \in(0,1)$. Since the metric (1) dominates the metric (5) on $N^{+}$, we see that $C^{p}$ is a closed subspace of $H^{p}$ for each $p \in(0,1)$. The spaces $C^{p}$ are closed under the conjugation operator, and the definition (1) implies that conjugation is isometric.

There are several alternative descriptions of the spaces $C^{p}$ :

Proposition 2. $C^{p}=R^{p}+i R^{p}$ for each $p \in(0,1)$.

Proof. The containment $R^{p}+i R^{p} \subseteq C^{p}$ is clear. If $f=a+i b$ belongs to $C^{p}$ (where the functions $a, b$ belong to $R^{+}$), then $\widehat{f}=a-i b$ belongs to $C^{+}$and satisfies $|f|=|\widehat{f}|$ a.e. on $\partial \mathbb{D}$. In particular, $C^{p}$ contains $\widehat{f}$, and hence $a$ and $b$ belong to $R^{p}$. Thus $f$ belongs to $R^{p}+i R^{p}$, which concludes the proof.

Proposition 3. $C^{p}=H^{p} \cap \overline{H^{p}}$ as subspaces of $L^{p}$ for each $p \in(0,1)$.

Proof. If $f$ belongs to $C^{p}$, then $\bar{f}=\widehat{f}$ a.e. on $\partial \mathbb{D}$ and $f$ belongs to $H^{p} \cap \overline{H^{p}}$. Conversely, if $f=I_{f} F$ belongs to $H^{p} \cap \overline{H^{p}}$, then there exists an inner function $I_{\widehat{f}}$ such that $I_{f} F=\overline{I_{\widehat{f}} F}$ a.e. on $\partial \mathbb{D}$. If $\widehat{f}$ denotes $I_{\widehat{f}} F$, then the functions $a=\frac{1}{2}(f+\widehat{f})$ and $b=\frac{1}{2 i}(f-\widehat{f})$ belong to $R^{p}$, and hence $f=a+i b$ belongs to $C^{p}$.

We remark that the algebra $C^{+}$can be used to obtain an explicit analog of Beurling's theorem for the backward shift operator

$$
f(z) \longmapsto \frac{f(z)-f(0)}{z}
$$

on $H^{p}$ for $p \in[1, \infty)$. We digress briefly on this application.

Let $\mathbb{D}_{e}$ denote the complement of the closed unit disk in the extended complex plane. If $f$ and $\tilde{f}$ are meromorphic functions on $\mathbb{D}$ and $\mathbb{D}_{e}$, respectively, with nontangential limiting values that agree almost everywhere on $\partial \mathbb{D}$, then $f$ and $\tilde{f}$ are called pseudocontinuations of one another. A meromorphic function on $\mathbb{D}_{e}$ is of bounded type if it is the quotient of bounded analytic functions on $\mathbb{D}_{e}$. The following remarkable theorem appears in 3 and relates pseudocontinuations to the backward shift operator.

Theorem (Douglas, Shapiro, Shields). A function $f \in H^{2}$ is noncyclic for the backward shift operator if and only if $f$ has a pseudocontinuation to $\mathbb{D}_{e}$ which is of bounded type.

More generally, this theorem holds for all $p \in(0, \infty)$ (see [2] for full details). If $f$ belongs to $H^{p}$ and has a meromorphic continuation to $\mathbb{D}_{e}$, then $f$ is trivially pseudocontinuable. The simplest nontrivial examples of pseudocontinuable functions are inner functions. If $\varphi$ is an inner function, then

$$
\tilde{\varphi}(z)=1 / \overline{\varphi(1 / \bar{z})}
$$


is a pseudocontinuation of $\varphi$ to $\mathbb{D}_{e}$ which is of bounded type. Functions with isolated branch points on $\partial \mathbb{D}$, such as $\sqrt{1+z}$, do not possess pseudocontinuations and are therefore cyclic. For more information on pseudocontinuations, see [10].

It is well known that the proper, nontrivial invariant subspaces for the backward shift operator on $H^{p}$ for $p \in[1, \infty)$ are precisely the subspaces $H^{p} \cap \varphi \overline{z H^{p}}$ where $\varphi$ is a nonconstant inner function [2]. The author showed in [5] that if $\zeta$ is a point on $\partial \mathbb{D}$ such that $\varphi$ has a nontangential limiting value of unit modulus at $\zeta$, then

$$
H^{p} \cap \varphi \overline{z H^{p}}=k_{\zeta} C^{+} \cap H^{p}
$$

where

$$
k_{\zeta}(z)=\frac{1-\overline{\varphi(\zeta)} \varphi(z)}{1-\bar{\zeta} z} .
$$

This representation, combined with Helson's formula (2), explains the phenomenon of pseudocontinuations. They arise from inner functions.

\section{The Riesz MAP}

Since $C^{p}$ is a closed subspace of $L^{p}$, the quotient $L^{p} / C^{p}$ is an $F$-space under the standard quotient metric. In other words, if $[f]$ denotes the equivalence class modulo $C^{p}$ of a function $f \in L^{p}$, then

$$
\|[f]\|_{p}^{p}:=\inf _{\sigma \in C^{p}}\|f-\sigma\|_{p}^{p}
$$

induces a translation-invariant metric $\rho([f],[g]):=\|[f]-[g]\|_{p}^{p}$ with respect to which $L^{p} / C^{p}$ is complete. Similarly, we can regard $H^{p} / C^{p}$ as a closed subspace of $L^{p} / C^{p}$ with respect to this metric.

Given a function $f \in L^{p}$, we may write $f=u+v$ where $u$ and $v$ belong to $H^{p}$ and $\overline{H^{p}}$, respectively. For $p \in(0,1)$ this follows from a result of Aleksandrov [1] (see also [2, p.117]), which we discuss below. It is easily seen that this decomposition is unique modulo $C^{p}$, and hence each equivalence class $[f]$ belonging to $L^{p} / C^{p}$ decomposes uniquely as $[f]=[u]+[v]$. We can therefore define the Riesz map $P: L^{p} / C^{p} \rightarrow H^{p} / C^{p}$ by setting $P[f]:=[u]$. This is an analog of the Riesz projection operator from $L^{p}$ to $H^{p}$ for $p \in(1, \infty)$. Indeed, if we regard equivalence classes as collections of boundary functions, then $P[f] \subset H^{p}$ and the Riesz map returns the "analytic part" of $[f]$.

The primary goal of this section is to show that the Riesz map is bounded in the sense that $\|P[f]\|_{p}^{p} \leq K_{p}\|[f]\|_{p}^{p}$ for some positive constant $K_{p}$ depending only on $p$. Our first task is to show that, given a function $f \in L^{p}$, we can choose $u \in H^{p}$ and $v \in \overline{H^{p}}$ in such a way that $f=u+v$ and that $\|u\|_{p}^{p}$ can be compared to $\|f\|_{p}^{p}$. Our proof of this fact is based largely on Aleksandrov's proof of the equality $L^{p}=H^{p}+\overline{z H^{p}}$. In fact, the only major difference is that we are careful to select and keep track of the constants that appear throughout his proof. By doing so we are able to obtain the desired estimate.

Proposition 4. If $f \in L^{p}$ for some $p \in(0,1)$, then for every $\epsilon>0$ there exist functions $u \in H^{p}$ and $v \in \overline{H^{p}}$ such that $f=u+v,\|u\|_{p}^{p} \leq K_{p}(1+\epsilon)\|f\|_{p}^{p}$, and $\|v\|_{p}^{p} \leq K_{p}(1+\epsilon)\|f\|_{p}^{p}$ where $K_{p}:=\left\|(1-z)^{-1}\right\|_{p}^{p}$.

Proof. Fix $\epsilon>0$, and let $c_{n}$ be a decreasing positive sequence such that $\sum_{n=0}^{\infty} c_{n}<\frac{\epsilon}{2}$. Since the trigonometric polynomials are dense in $L^{p}$, there exists a sequence $q_{n}$ of 
trigonometric polynomials such that

$$
\left\|f-q_{n}\right\|_{p}^{p} \leq c_{n}\|f\|_{p}^{p}
$$

Define another sequence of trigonometric polynomials $p_{n}$ by setting $p_{0}=q_{0}$ and $p_{n}=q_{n}-q_{n-1}$ for $n \geq 1$. This new sequence satisfies the inequalities

$$
\begin{aligned}
\left\|p_{0}\right\|_{p}^{p} & \leq\left(1+c_{0}\right)\|f\|_{p}^{p}, \\
\left\|p_{n}\right\|_{p}^{p} & \leq\left(c_{n}+c_{n-1}\right)\|f\|_{p}^{p}, \quad n \geq 1 .
\end{aligned}
$$

The sum $\sum_{n=0}^{\infty} p_{n}$ converges in $L^{p}$ since

$$
\begin{aligned}
\sum_{n=0}^{\infty}\left\|p_{n}\right\|_{p}^{p} & \leq\left(1+c_{0}\right)\|f\|_{p}^{p}+\sum_{n=1}^{\infty}\left(c_{n}+c_{n-1}\right)\|f\|_{p}^{p} \\
& =\left(1+2 \sum_{n=0}^{\infty} c_{n}\right)\|f\|_{p}^{p} \\
& <(1+\epsilon)\|f\|_{p}^{p} .
\end{aligned}
$$

For each $\zeta \in \partial \mathbb{D}$, let $m_{n}>\operatorname{deg} p_{n}$ and define the functions

$$
u_{n}^{\zeta}(z)=\frac{z^{m_{n}} p_{n}(z)}{z^{m_{n}}-\zeta}, \quad v_{n}^{\zeta}(z)=\frac{z^{-m_{n}} p_{n}(z)}{z^{-m_{n}}-\bar{\zeta}} .
$$

Since $p_{n}$ is a trigonometric polynomial of degree smaller than $m_{n}$, the functions $u_{n}^{\zeta}$ and $v_{n}^{\zeta}$ belong to $H^{p}$ and $\overline{H^{p}}$, respectively. For each fixed $\zeta$ these functions satisfy $u_{n}^{\zeta}+v_{n}^{\zeta}=p_{n}$ and $\left|u_{n}^{\zeta}\right|=\left|v_{n}^{\zeta}\right|$ on $\partial \mathbb{D}$. Since $(1-z)^{-1}$ belongs to $H^{p}$ for each $p \in(0,1)$, Fubini's theorem implies that

$$
\int_{|\zeta|=1}\left\|u_{n}^{\zeta}\right\|_{p}^{p} d m(\zeta)=\left\|(1-z)^{-1}\right\|_{p}^{p}\left\|p_{n}\right\|_{p}^{p}
$$

and

$$
\int_{|\zeta|=1}\left\|v_{n}^{\zeta}\right\|_{p}^{p} d m(\zeta)=\left\|(1-z)^{-1}\right\|_{p}^{p}\left\|p_{n}\right\|_{p}^{p}
$$

In particular, there exists a $\zeta$ such that the inequalities

$$
\left\|u_{n}^{\zeta}\right\|_{p}^{p} \leq K_{p}\left\|p_{n}\right\|_{p}^{p}, \quad\left\|v_{n}^{\zeta}\right\|_{p}^{p} \leq K_{p}\left\|p_{n}\right\|_{p}^{p}
$$

hold. For this value of $\zeta$, we define $u_{n}:=u_{n}^{\zeta}$ and $v_{n}:=v_{n}^{\zeta}$.

In light of the inequality $\sum_{n=0}^{\infty}\left\|p_{n}\right\|_{p}^{p}<(1+\epsilon)\|f\|_{p}^{p}$, the series $\sum_{n=0}^{\infty} u_{n}$ and $\sum_{n=0}^{\infty} v_{n}$ converge in $L^{p}$ to functions $u \in H^{p}$ and $v \in \overline{H^{p}}$ satisfying

$$
\|u\|_{p}^{p} \leq K_{p}(1+\epsilon)\|f\|_{p}^{p}, \quad\|v\|_{p}^{p} \leq K_{p}(1+\epsilon)\|f\|_{p}^{p} .
$$

Moreover,

$$
u+v=\sum_{n=0}^{\infty} p_{n}=f,
$$

as required.

We are now in a position to prove the continuity of the Riesz map.

Proposition 5. The Riesz map is bounded for each $p \in(0,1)$. For every $f \in L^{p}$ we have $\|P[f]\|_{p}^{p} \leq K_{p}\|[f]\|_{p}^{p}$. 
Proof. Let $f$ be an arbitrary function in $L^{p}$. For each $\epsilon>0$ pick $f_{\epsilon} \in[f]$ such that

$$
\left\|f_{\epsilon}\right\|_{p}^{p} \leq(1+\epsilon)^{\frac{1}{2}}\|[f]\|_{p}^{p}
$$

By Proposition 4, there exist functions $u_{\epsilon}$ and $v_{\epsilon}$ in $H^{p}$ and $\overline{H^{p}}$, respectively, such that $f_{\epsilon}=u_{\epsilon}+v_{\epsilon}$ and

$$
\left\|u_{\epsilon}\right\|_{p}^{p} \leq K_{p}(1+\epsilon)^{\frac{1}{2}}\left\|f_{\epsilon}\right\|_{p}^{p}
$$

Since each $u_{\epsilon}$ belongs to $P\left[f_{\epsilon}\right]=P[f]$ we have

$$
\|P[f]\|_{p}^{p} \leq\left\|u_{\epsilon}\right\|_{p}^{p} \leq K_{p}(1+\epsilon)\|[f]\|_{p}^{p} .
$$

The desired inequality follows by letting $\epsilon$ tend to zero.

Since $C^{p}=\mathbb{C}$ for $p \geq 1$, we can regard the Riesz projection operator on $H^{p}$ for $p \in(1, \infty)$ as an operator on the quotient $H^{p} / C^{p}=H^{p} / \mathbb{C}$. Hence the Riesz map on $H^{p} / C^{p}$ for $p \in(0,1)$ and the Riesz projection operator on $H^{p}$ for $p \in(1, \infty)$ are two manifestations of the single concept of "analytic projection."

It is well known that the Riesz projection operator is not well-defined on $L^{1}$. Indeed, there are no bounded projections from $L^{1}$ onto $H^{1}$. Nevertheless, Proposition 5 and the preceding discussion indicate that a reasonable version of the Riesz operator exists for $p \in(0,1)$. Examining the proof of Proposition 4, we see that the constants $K_{p}$ grow without bound as $p$ increases to 1 since $(1-z)^{-1}$ does not belong to $H^{1}$. Thus we encounter problems with "analytic projection" as we approach $p=1$ from above and below.

\section{The $\mathcal{T}$-TRANSFORM}

In this section we introduce a certain integral transform and consider its relationship to $C^{p}$. Define the $\mathcal{T}$-transform $\mathcal{T}_{\mu}$ of a finite measure $\mu$ on $\partial \mathbb{D}$ to be

$$
\mathcal{T}_{\mu}(z):=\int_{\partial \mathbb{D}} T(z \bar{\zeta}) d \mu(\zeta)
$$

where $T$ is the linear fractional transformation defined by Equation (31). Note the similarity between the $\mathcal{T}$-transform and the Herglotz integral of $\mu$.

If $\mu$ is a positive singular measure on $\partial \mathbb{D}$, then the imaginary part of $\mathcal{T}_{\mu}$ is positive on $\mathbb{D}$ and vanishes a.e. on $\partial \mathbb{D}$ by a standard argument. Specifically, one views the imaginary part of $\mathcal{T}_{\mu}$ as the Poisson-Stieltjes integral (modulo some constant factors) of a bounded nondecreasing function of bounded variation whose derivative vanishes a.e. on $\partial \mathbb{D}$ (since $\mu$ is singular) [4, Ch.1]. In particular, there exists a nonconstant inner function $\varphi_{\mu}$ such that

$$
\mathcal{T}_{\mu}=T\left(\varphi_{\mu}\right) .
$$

If $\varphi$ is a nonconstant inner function, then $\operatorname{Im} T(\varphi)$ is positive on $\mathbb{D}$ and vanishes a.e. on $\partial \mathbb{D}$. Hence $\operatorname{Im} T(\varphi)$ can be recovered as the imaginary part of the $\mathcal{T}$ transform of some positive singular measure $\mu$ on $\partial \mathbb{D}$. Passing to the analytic completion we find that $T(\varphi)=\mathcal{T}_{\mu}+c$ for some real constant $c$. Evaluating at $z=0$ we find that $T(\varphi(0))=c+i\|\mu\|$ where $\|\mu\|$ denotes the total variation norm of $\mu$. From the definition of $T$ we see that $c=0$ precisely when $\varphi(0)$ is purely imaginary. Therefore a nonconstant inner function $\varphi$ is the $\mathcal{T}$-transform of a positive singular measure if and only if $\varphi(0)$ is purely imaginary. 
Equation (6) furnishes a bijection between positive singular measures on $\partial \mathbb{D}$ and nonconstant inner functions whose value at the origin is purely imaginary. In particular we have $T\left(\varphi_{\mu}(0)\right)=\|\mu\|$.

If $\mu$ is a real finite singular measure, then write $\mu=\mu^{+}-\mu^{-}$where $\mu^{+}$and $\mu^{-}$ are positive singular measures such that $\mu^{+} \perp \mu^{-}$. Since the $\mathcal{T}$-transform is clearly additive, there exist nonconstant inner functions $\varphi_{\mu^{+}}$and $\varphi_{\mu^{-}}$such that

$$
\mathcal{T}_{\mu}=T\left(\varphi_{\mu^{+}}\right)-T\left(\varphi_{\mu^{-}}\right) .
$$

Therefore the $\mathcal{T}$-transform of any real finite singular measure belongs to $R^{p}$ for every $p \in(0,1)$ and has the form (7). The representation of $\mathcal{T}_{\mu}$ as the difference of the $\mathcal{T}$-transforms of two positive singular measures is not unique unless we also require that the two measures be mutually singular.

Now let $\mu_{1}$ and $\mu_{2}$ be two positive singular measures on $\partial \mathbb{D}$ and define $\varphi_{1}:=\varphi_{\mu_{1}}$ and $\varphi_{2}:=\varphi_{\mu_{2}}$ to be their corresponding inner functions. There is a divisibility relationship between $\varphi_{1}, \varphi_{2}$ and the inner function $\varphi_{\mu}$ corresponding to a convex combination of $\mu_{1}$ and $\mu_{2}$.

Proposition 6. If $\mu=\lambda_{1} \mu_{1}+\lambda_{2} \mu_{2}$ where $\lambda_{1}, \lambda_{2} \in[0,1]$ and $\lambda_{1}+\lambda_{2}=1$, then $\mathcal{T}_{\mu}=T\left(\varphi_{\mu}\right)$ where the inner function $\varphi_{\mu}$ is given by

$$
\varphi_{\mu}=i \frac{\varphi_{1} \varphi_{2}-i \lambda_{1} \varphi_{1}-i \lambda_{2} \varphi_{2}}{1+\lambda_{2} \varphi_{1}+\lambda_{1} \varphi_{2}} .
$$

Furthermore, the greatest common divisor of $\varphi_{1}$ and $\varphi_{2}$ divides $\varphi_{\mu}$.

Proof. The formula for $\varphi_{\mu}$ follows from the calculation of $\lambda_{1} T\left(\varphi_{1}\right)+\lambda_{2} T\left(\varphi_{2}\right)$. Since $1+\lambda_{2} \varphi_{1}+\lambda_{1} \varphi_{2}=\lambda_{2}\left(1+\varphi_{1}\right)+\lambda_{1}\left(1+\varphi_{2}\right)$, we see that the denominator of the expression in (8) is the sum of two outer functions assuming values in the right half plane and is therefore outer. This implies the second statement.

If $\mu$ is a finite complex singular measure on $\partial \mathbb{D}$, then we may write $\mu=\mu_{1}+i \mu_{2}$ where $\mu_{1}$ and $\mu_{2}$ are finite real singular measures. Linearity and Equation (7) tell us that $\mathcal{T}(\mu)$ belongs to $C^{p}$ for each $p \in(0,1)$. Moreover, the $\mathcal{T}$-transform embeds the nonseparable space $M_{s}$ of singular measures in $C^{p}$ for each $p \in(0,1)$ in a way that is compatible with the conjugation operator.

Proposition 7. The $\mathcal{T}$ transform maps conjugate measures to conjugate functions, in the sense defined in Section 3 .

$$
\mathcal{T}_{\bar{\mu}}=\widehat{\mathcal{T}_{\mu}}
$$

\section{REFERENCES}

[1] Aleksandrov, A.B, Essays on non-locally convex Hardy spaces, "Complex Analysis and Spectral Theory," Lecture Notes in Mathematics 864, V. P. Havin and N. K. Nikol'skii (editors), Springer-Verlag, 1980. MF0643380 (84h:46066)

[2] Cima, J.A., Ross, W.T., The Backward Shift on the Hardy Space, American Mathematical Society, 2000. MR,1761913 (2002f:47068)

[3] Douglas, R.G., Shapiro, H.S., Shields, A.L., Cyclic vectors and invariant subspaces for the backward shift operator, Ann. Inst. Fourier (Grenoble) 20 no. 1 (1970), 37-76. MR0270196 (42:5088)

[4] Duren, P.L., Theory of $H^{p}$ Spaces, Pure and Appl. Math., Vol 38, Academic Press, New York, 1970. MR0268655 (42:3552)

[5] Garcia, S.R., Conjugation, the backward shift, and Toeplitz kernels, (To appear: J. Operator Theory). 
[6] Garcia, S.R., Sarason, D., Real outer functions, Indiana Univ. Math. J. 52 (2003), 13971412. MR 2021044

[7] Helson, H., Large analytic functions II. Analysis and Partial Differential Equations, C. Sadosky (ed.), Marcel Dekker, New York (1990), pp. 217-220. MR.1044789|(92c:30039)

[8] Helson, H., Sarason, D., Past and future, Math. Scand. 21 (1967), 5-16. MR0236989 $(38: 5282)$

[9] Neuwirth, J., Newman, D.J., Positive $H^{\frac{1}{2}}$ functions are constants, Proc. Amer. Math. Soc. 18 (1967), 968. MR0213576 (35:4436)

[10] Ross, W.T., Shapiro, H.S., Generalized Analytic Continuation, University Lecture Series, Volume 25, American Mathematical Society, Providence, 2002. MR,1895624 (2003h:30003)

Department of Mathematics, University of California at Santa Barbara, Santa Barbara, California 93106-3080

E-mail address: garcias@math.ucsb.edu

$U R L:$ http://math.ucsb.edu/ ${ }^{\text {garcias }}$ 